\title{
Lyme Borreliosis with Opthalmologic Presentation
}

\author{
Ayse Gultekingil Keser ${ }^{1}$, Rezan Topaloglu' ${ }^{2}$,Mehmet Orhan ${ }^{3}$, Yelda Bilginer ${ }^{2}$, Esra Baskin ${ }^{4}$, Yonca Akova ${ }^{5}$ \\ ${ }^{1}$ Department of Pediatrics, Hacettepe University Faculty of Medicine, Ankara, Turkey; ${ }^{2}$ Department of Pediatric Nephrology and \\ Rheumatology, Hacettepe University Faculty of Medicine, Ankara, Turkey; ${ }^{3}$ Department of Opthalmology, Hacettepe University \\ Faculty of Medicine, Ankara, Turkey; ${ }^{4}$ Department of Pediatric Nephrology and Rheumatology, Baskent University Faculty of \\ Medicine, Ankara, Turkey; ${ }^{5}$ Department of Opthalmology, Baskent University Faculty of Medicine, Ankara, Turkey. \\ Email: aysegultek@yahoo.com
}

Received August $3^{\text {rd }}, 2012$; revised September $12^{\text {th }}, 2012$; accepted September $20^{\text {th }}, 2012$

\begin{abstract}
Lyme disease is a tick-borne multisystemic disease with multiple clinical presentations including rheumatological conditions. We present a 12 year old girl complaining of fever, arthralgia and redness in her eyes which was found to be the result of panuveitis. Borrelia antibodies were positive and she received 21-day treatment regimen with Ceftriaxon with resolution of all complaints at the end of treatment. Lyme disease classically occurs in three stages starting with erythema migrans, however atypical presentations skipping this stage and presenting with opthalmologic involvement in form of uveitis can be seen.
\end{abstract}

Keywords: Lyme; Borreliosis; Uveitis

\section{Introduction}

Lyme disease is one of the most common tick-borne infectious diseases [1]. The incidence varies between 0.3 - 155/100,000, but clinically overt disease occur only $0.3 \%-1.4 \%$ of tick bites [1,2]. The causative pathogen is Borrelia burgdoferi sensu lato and discovery of the pathogen is a firm way of diagnosing the disease [1]. Lyme disease is a multisystemic disorder, clinical presentation takes place in three stages (early localized, early disseminated and late disseminated), starting with a skin lesion after tick bite, heart and nervous system involved later, followed by joint, eye, nervous system and skin, however complete clinical presentation is extremely rare $[2,3]$. Symptoms may mimick many musculoskeletal, neurologic and rheumatologic conditions [2]. Hereby we present an atypical presentation of lyme disease with papillitis and uveitis.

\section{Case Presentation}

A 12-year-old girl patient admitted to our clinic with a history of eighteen days of fever and arthralgia of ankles. She did not have any rash accompanying fever but had redness in her eyes, mild myalgia, nausea and vomitting . She did not have similar symptoms before she had been followed for ten days at another center, complete blood cell count and biochemical markers were within normal limits but sedimentation rate and C-Reactive Protein (CRP) were elevated. (sedimentation: $69 \mathrm{~mm} / \mathrm{h}$,
CRP: 170.6 mg/L). Viral markers were negative, Brucella agglutination was negative, Anti Nuclear Antibodies (AN-A), c-ANCA, p-ANCA were negative, anti dsDNA titer were within normal limits. Eye examination showed severe panuveitis and papillitis (Figures 1(a) and (b)).

Cranial tomography was normal. Echocardiography showed no pericardial or pleural effusions. Ceftriaxon therapy was started for the treatment of an infection of unknown origin and steroid eye drops for uveitis. After ten days of ceftriaxon treatment the patient admitted to our clinic with complaints of fatigue, myalgia and still elevated acute phase reactans.

On physical examination, she did not have any kind of rash and there was no sign of arthritis. Blood pressure was within normal limits. Opthalmologic examination revealed continuous but milder papilitis and panuveitis compared to previous examination. Sedimentation rate was still high ( $67 \mathrm{~mm} / \mathrm{h})$, ANA was $1 / 80$ stained in nucleolar pattern and anti ds DNA was elevated (194 IU/ $\mathrm{mL}$ ). C3 and C4 levels were normal. Anticardiolipin and anti phospholipid antibodies were negative. Urinanalysis showed no proteiuria. Because of continous papillitis, panuveitis and history of fever, arthralgia, myalgia, the tests for infectious etiology were extended.

Borrelia antibodies were studied by enzyme linked immunoassay (ELISA) test and IgM was found to be > 200 IU and IgG was negative. Result of the test was confirmed with western blot analysis. She was diagnosed as Lyme Borreliosis with panuveitis and papillitis and 


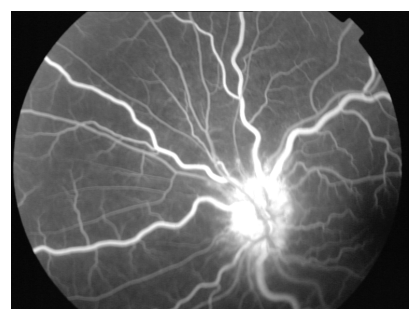

(a)

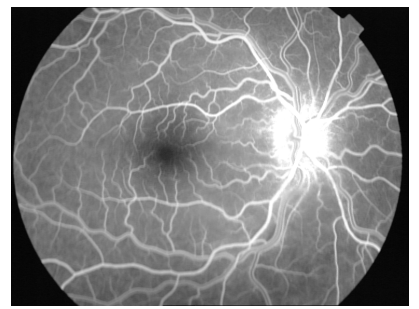

(b)

Figure 1. (a) Papillitis with mild papilledema and increased venous tortuosity in fundus, floresein angiography; (b) Papillitis with mild papilledema and increased venous tortuosity in fundus, floresein angiography.

ceftriaxon therapy was started again and continued until twenty day treatment regimen was completed.

She was afebrile at the end of treatment, arthralgia and myalgia were resolved. Acute phase reactants were within normal limits (ESR $12 \mathrm{~mm} / \mathrm{hr}$ and CRP < 0.5) ANA and anti ds DNA were negative. In opthalmologic examination, there was no sign of uveitis and papillitis (Figure 2) Borrelia IgM became negative. She was followed for 6 months. She did not have any complaints in that period. She never had proteinuria. Her ANA was 1/160 again but anti ds DNA was negative and acute phase reactants were within normal limits at 6 months. She has been clinically healthy since then.

\section{Discussion}

Our patient was in the risk group of borreliosis as most cases of borreliosis are seen in people aged 5 - 14 years and 50 - 59 years [4], 20,000 new cases reported every year and 30\% cases occur in children [5]. Clinical course in most children is milder and shorter than adults [5].

After Ixodes tick bite,following localized skin rash, within days to weeks spirochetes disseminate resulting in the presence of B.burgdoferi in blood, cerebrospinal fluid, heart, retina, brain, muscle, bone, spleen, liver and meninges of patients leading to a variety of symptoms $[4,6]$. Stage 1 of disease occurs from days to weeks after tick bite and characterized by typical rash of Erythema migrans [1]. Stage 2 of disease occurs from weeks to 6 months and characterized by radiculitis, meningitis, peripheral facial nerve palsy, encephalitis, myelitis, myalgia, arthritis, myositis, iritis, myocarditis, pericarditis and multiple erythemas [1]. Stage 3 of disease occurs after 6

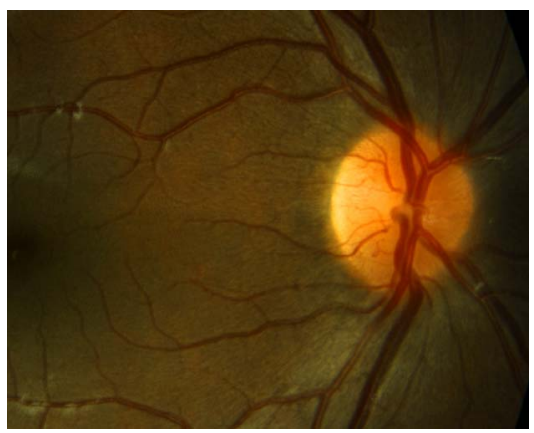

Figure 2. Healthy optic disc after treatment.

months and characterized by encephalitis, encephalo-myelitis, serebral arteritis, polyneuropathy, mono or oligoarthritis and acrodermatitis chronica [1].

About 30\% - 70\% patients have constitutional complaints including malaise and fatigue, headache, short lasting migratory arthralgias and myalgias, low grade fever and lymphadenopathy [1-4]. Rheumatological manifestations include musculoskeletal pain and short attacks of frank arthritis [4]. Migrating transitory musculoskeletal pain is common in Lyme disease, $1 / 5$ of patients have short episodes of pain limited to joints with no objective sign related to joints [2-5].

Acute neuroborreliosis is diagnosed only in $10 \%-15 \%$ of patients, it develops after weeks to months [2,4,5]. Early invasion into central nervous system can occur within 2 weeks however only half of these patients have CNS symptoms during this period [6]. Bacterial content in central nervous sytem is often low, bacterial contituents are released in small quantities over an extended period of time [1]. Neurologic involvement presents as inflamation in subarachnoid and perineural tissue [6]. Most typical manifestations are lymphocytic meningitis, radiculoneuritis, cranial neuritis, in children most common neurological sign is facial nerve palsy $[2,4,5,7,8]$. Sleep disturbance and papiledema associated with increased intracranial pressure are less common manifestations [5].

Ocular neuroborreliosis can be seen in every stage but most frequently in second and third stages [5,7,9]. Various ocular symptoms including pain, visual impairment, photophobia, diplopia and lack of accomadation may be seen [9]. Opthalmologic changes include conjuctivitis, optic neuritis and all varieties of uveitis, most commonly posterioual impairment [10]. In any patient with uveitis, infectious cause should be ruled out first, differential diagnosis include herpes, toxoplasma, tuberculosis, Lyme borreliosis and others [11]. Most common form is intermediate uveitis, commonly associated with iridocyclitis [9]. Anterior uveitis is infrequent, it may be associated with papillitis [9]. Only few reports concern panuveitis but it may result in irreversible visual loss [9]. Macular edema and vasculitis are most frequent findings of posterior segment inflammation, cotton wool 
spots could also be a sign of Lyme retinitis [7-9].

Ocular inflammation occuring in an endemic zone, report of contact with a tick, previous history of erythema migrans, positive serology with presence of IgM in early stage or IgG in late stage raise the suspicion of Lyme disease [9]. Production of IgM starts 2 - 4 weeks after infection and peaks at 6 - 8 weeks [4], therefore detection of specific antibody in serum in early phase of infection is neither necessary nor sufficient, but if infection lasts longer than 8 weeks, antibodies can be detected in serum [1]. Whenever disseminated borreliosis is suspected on clinical grounds, serologic examination should be performed immediately and repeated 3 weeks later if results are negative [1]. Borrelia specific IgM or IgG antibodies are found in serum of $>90 \%$ patients with neurological manifestations [1]. Detection of serum antibodies is based on two step approach, first highly sensitive ELISA test should be used as a screening test, positive or borderline results should be confirmed with western blot or immunoblot assay $[1,4,5]$.

Antibiotic treatment not only shortens duration of illness but also prevents complications and chronic infections [1]. For patients with Lyme neuroborreliosis penicillin, cefotaxime, ceftriaxone, doxycycline are effective, ceftriaxone or cefotaxime for $14-21$ days is recommended because of their low MIC values [1,2]. Ocular manifestations are considered to be severe, so ceftriaxone or cefotaxime therapy is recommended for these conditions [4]. When disease is treated properly, recurrence is rare, but clinical manifestations take long time to resolve even after the pathogen has been eliminated [1]. Stage 2 neuroborreliosis resolves completely after 12 months in $95 \%$ of patients [1].

Course of disease may skip any individual stage for example a patient with neuroborreliosis does not need to have erythema migrans in past [1], so was the case in our patient. She did not have a history of tick bite or erythema migrans which made the clinical diagnosis rather difficult. She only had nonspecific symptoms of fever, malaise, arthralgia and myalgia. However, she also had papiledema, papillitis and panuveitis which do not have an underlying etiology. Her fever, arthralgia, myalgia and her panuveitis partially benefited from nonspecific ceftriaxone therapy leading us to suspect Lyme borreliosis. Therefore ELISA study was done and result was positive for borreliosis and confirmed with western blot. Her anti ds DNA level was high in one study but she never had any other symptoms of systemic lupus erythematosus, and in a short period of time it returned to normal so we concluded that this result may be due to cross reaction. As a result ceftriaxone therapy was completed to 21 day course of treatment as in guidelines and all symptoms of the patient resolved at the end of treatment without any need for further therapy and this fact also supported our diagnosis.
Our patient had atypical signs and symptoms of borreliosis that diagnosed with opthalmologic problems. Therefore, it should be kept in mind that lyme borreliosis may have many different presentations and it should be considered in differential diagnosis of unexplained clinical pictures.

\section{REFERENCES}

[1] R. Nau, H. J. Christen and H. Eiffert, "Lyme DiseaseCurrent State of Knowledge," Deutches Arzteblatt International, Vol. 106, No. 5, 2009, pp. 72-81.

[2] J. Hytönen, P. Hartiala, J. Oksi and M. K. Viljanen, "Borreliosis: Recent Research, Diagnosis, and Management," Scandinavian Journal of Rheumatology, Vol. 37, No. 3, 2008, pp. 161-172. doi:10.1080/03009740801978897

[3] F. Strle and G. Stanek, "Clinical Manifestations and Diagnosis of lyme borreliosis," Current Problems in Dermatology, Vol. 37, 2009, pp. 51-110. doi:10.1159/000213070

[4] S. Schnarr, J. K. Franz, A. Krause and H. Zeidler, "Infection and Musculoskeletal Conditions: Lyme Borreliosis," Best Practice \& Research Clinical Rheumatology, Vol. 20, No. 6, 2006, pp. 1099-1118. doi:10.1016/j.berh.2006.08.006

[5] P. Hildenbrand, D. E. Craven, R. Jones and P. Nemeskal, "Lyme Neuroborreliosis: Manifestations of a Rapidly Emerging Zoonosis,” American Journal of Neuroradiology, Vol. 30, No. 6, 2009, pp. 1079-1087. doi:10.3174/ajnr.A1579

[6] B. A. Fallon, E. S. Levin, P. J. Schweitzer and D. Hardesty, "Inflammation and Central Nervous System Lyme Disease," Neurobiology of Disease, Vol. 37, No. 3, 2010, pp. 534541. doi:10.1016/j.nbd.2009.11.016

[7] A. J. Klaeger and C. P. Herbort, "Cotton Wool Spots as Possible Indicators of Retinal Vascular Pathology in Ocular Lyme Borreliosis,” International Ophthalmology, Vol. 30, No. 5, 2010, pp. 599-602. doi:10.1007/s10792-008-9268-5

[8] M. Baumann, R. Birnbacher, J. Koch, R. Strobl and K. Rostásy, "Uncommon Manifestations of Neuroborreliosis in Children,” European Journal of Paediatric Neurology, Vol. 14, No. 3, 2010, pp. 274-277. doi:10.1016/j.ejpn.2009.08.003

[9] P. Mora and A. Carta, "Ocular Manifestations of Lyme Borreliosis in Europe,” International Journal of Medical Science, Vol. 6, No. 3, 2009, pp. 124-125. doi:10.7150/ijms.6.124

[10] A. de-la-Torre, C. A. López-Castillo, J. C. Rueda, R. D. Mantilla, J. E Gómez-Marín and J. M. Anaya, "Clinical Patterns of Uveitis in Two Ophthalmology Centres in Bogota, Colombia," Clinical \& Experimental Ophthalmology, Vol. 37, No. 5, 2009, pp. 458-466. doi:10.1111/j.1442-9071.2009.02082.x

[11] M. Khairallah, S. P. Chee, S. R. Rathinam, S. Attia, and V. Nadella, "Novel Infectious Agents Causing Uveitis," International Ophthalmology, Vol. 30, No. 5, 2010, pp. 465-483. doi:10.1007/s10792-009-9319-6 